\title{
Corticosterone, Avoidance of Novelty, Risk-Taking and Aggression in a Wild Bird: No Evidence for Pleiotropic Effects
}

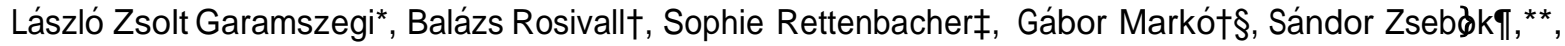 \\ Eszter Szöllゆsi†, Marcel Eens††, Jaime Potti* \& János Török† \\ * Department of Evolutionary Ecology, Estación Biológica de Doñana-CSIC, Seville, Spain \\ $†$ Behavioural Ecology Group, Department of Systematic Zoology and Ecology, Eötvös Loránd University, Budapest, Hungary \\ ‡ Department of Biomedical Sciences, Biochemistry, University of Veterinary Medicine Vienna, Vienna, Austria \\ $\S$ Department of Plant Pathology, Corvinus University of Budapest, Budapest, Hungary \\ I Sensory Ecology Group, Max Planck Institute for Ornithology, Seewiesen, Germany \\ ** MTA-ELTE-MTM Ecology Research Group, Budapest, Hungary \\ ††Department of Biology, University of Antwerp Wilrijk, Belgium
}

Correspondence

László Zsolt Garamszegi, Department of Evolutionary Ecology, Estación Biológica de Doñana-CSIC, c/Americo Vespucio, s/n, 41092, Sevilla, Spain.

E-mail: laszlo.garamszegi@ebd.csic.es

Received: January 26, 2012

Initial acceptance: April 2, 2012

Final acceptance: April 11, 2012

(L. Fusani)

\begin{abstract}
Certain inherent characteristics of individuals can determine both physiological and behavioural responses to environmental challenges, which could drive a correlation between levels of corticosterone (CORT), the most important stress hormone and behavioural profiles. Therefore, CORT level may mediate consistent behaviours along the shy/bold continuum, and thus, it could serve as a pleiotropic basis for behavioural syndromes. Moreover, behavioural responses to environmental challenges may have consequences for CORT concentrations, which would also result in a correlation between physiology and behaviours even without requiring pleiotropic mechanisms. Accordingly, we investigated the relationship between CORT and behaviour in free-living male collared flycatchers, Ficedula albicollis, using recently developed field assays. More specifically, we characterised novel object avoidance, intraspecific aggression and risk-taking in males and related these correlated behaviours to the concentration of CORT metabolites in droppings measured by enzyme immunoassay. Individuals with higher levels of excreted CORT metabolites had no consistently higher or lower behavioural scores along the shy/ bold spectrum, as avoidance of novelty, aggression and risk-taking were not systematically related to CORT metabolite concentrations in the same direction. Moreover, environmental challenges owing to the presence of a novel object, territorial intruder and a potential predator caused no elevation in the level of CORT metabolites. Therefore, we did not find correlative evidence for CORT driving correlated behaviours through pleiotropic effects or for particular behaviours during courtship causing elevation in CORT levels.
\end{abstract}

\section{Introduction}

One strategy of animals to cope with challenges in their physical and social environment is to display generally consistent behavioural responses along the bold/shy (or fast/slow or active/passive or proactive/ reactive) continuum to different stimuli (Koolhaas et al. 1999; Gosling 2001; Groothuis \& Carere 2005), which often results in the correlation between behaviours. This phenomenon emerges because individuals with inherently bold characteristics will generally behave actively in different ecological or social situations, while shy conspecifics will be less active in these situations. For example, animals behaving aggressively in a social challenge will also respond with high activity to the presence of a predator thus will appear 
as risk takers shaping a correlation between aggression and risk-taking. As such correlated behaviours - so called behavioural syndromes - may drive life-history trade-offs and ultimately determine fitness, they have high ecological and evolutionary relevance, and they have recently received considerable interest (Sih et al. 2004a,b; Réale et al. 2007; Herczeg \& Garamszegi 2012).

Understanding the physiological basis of correlated behaviours is elementary for being able to make evolutionary deductions. One of the key questions is whether behavioural syndromes arise from a shared proximal link between behaviours, for example, the effect of pleiotropic hormones or genes that govern different behaviours (Bell 2005). A common physiological control acting as a constraint would imply that correlated behaviours cannot evolve independently if selection conserves them mechanistically coupled (Arnold 1992; Lande 1992). When one type of behaviour is favoured it would have consequences for an entire suite of other behaviours that also rely on the same physiological basis (Stamps 1991; Ketterson \& Nolan 1999). Pleiotropic effects may be manifested through a single mediating factor (such as a hormone or gene), which is activated in response to stimuli from various ecological situations and which triggers different tissues or cells that generate or maintain consistent behaviours. Pleiotropy may also occur via the physiological control of responsiveness, which determines the degree by which individuals perceive different stimuli as a threat (Sapolsky 1994; Koolhaas et al. 2001; Wolf et al. 2008).

An obvious marker that can reflect the importance of the concerted physiological regulation of behavioural syndromes is corticosterone (CORT), a stress hormone that is intensively investigated in birds. Physiological responses to stress stimuli from the physical or social environment involve the regulation of fuel metabolism through hormone-mediated mechanisms, while behaviours such as locomotion or foraging may also change in response to high CORT levels (Silverin 1997; Belthoff \& Dufty 1998; Saldanha et al. 2000). Alternatively, individual differences in the performance of behaviours may be determined by innate (i.e. shy/bold or proactive/reactive) characteristics that simultaneously affect behavioural and physiological responses to various environmental stimuli and that would raise an indirect relationship between baseline CORT concentrations and many behaviours (Cockrem 2007). Individuals systematically differ in both baseline and stress-induced changes in CORT levels reflecting that they inherently cope differentially with stress (Satterlee \& Johnson 1988; Cockrem
\& Silverin 2002b; Odeh et al. 2003; Kralj-Fiser et al. 2007; Stöwe et al. 2010; Lendvai et al. 2011). The repeatable and heritable individual variation in CORT levels in response to environmental changes suggests a link with behavioural syndromes, which also rely on individual consistency and determine how individuals cope with environmental demands at the behavioural level (Carere et al. 2003; Kralj-Fiser et al. 2007; Schoech et al. 2009). A more direct evidence for such a link has been demonstrated in the great tit Parus major, in which baseline and stress-induced CORT excretion was different between groups of nestlings that originated from lines that had been selected for slow and fast exploration (Stö we et al. 2010).

Additionally, variation in stress-induced CORT levels cannot only be the cause, but also the consequence of behavioural variations, if environmental stimuli or solicited behaviours per se affect stress physiology. During performance, individuals may expose themselves to different threats that subsequently stimulate physiological stress and CORT production. For example, intraspecific aggression can be considered as an important stress factor (Koolhaas et al. 1997), thus aggressive and bold phenotypes may be subject to repeated increases in plasma CORT and may repeatedly experience situations of enhanced CORT secretion during the breeding season (Mateos

2005). The engagement in territory disputes may favour the elevation of CORT concentrations as a response to the social stress that a male experiences during aggressive interactions. An immediate increase in CORT levels in this context may help mobilising energy reserves by triggering gluconeogenesis in resident males needed to fight with other males and defend the territory (Wingfield \& Romero 2000; Orchinik et al. 2002). Moreover, high hormone levels maintained during active intrasexual conflicts may be adaptive as it might be physiological basis to tolerate injuries obtained, as it has been suggested in mice (Avitsur et al. 2001). A similar argument could be applied to other components of behavioural syndromes, as active and passive behaviours in novel habitats or during the presence of predator can also involve different threats and thus generate different levels of stress.

Accordingly, several avian studies have revealed a relationship between CORT and behavioural traits regarded as components of behavioural syndromes (see reviews in Carere et al. 2010; Cockrem 2007; Koolhaas et al. 1999). The general tendency emerging from these studies seems that high CORT levels (either baseline or stress-induced) are associated with shy/slow/passive/reactive behavioural types, while 
low hormone levels are typical for the bold/fast/ active/proactive profiles. However, this does not seem to be an unbreakable rule, as some studies found the opposite trend or did not detect a correlation between CORT and behaviour (e.g. Jones et al. 1995; Korte et al. 1997; Mettke-Hofmann et al. 2006; Martins et al. 2007; Kralj-Fiser et al. 2010). Hence, the importance of CORT in mediating correlations among behaviours is still unclear. From the available evidence, it is difficult to decide whether CORT levels determine how animals behave in response to an environmental challenge or behavioural reactions have consequences for hormone concentration. Finally, it remains an open question whether baseline- or stress-induced CORT levels play role in these processes.

Studies on behavioural syndromes in completely natural circumstances are still scarce, especially in the context of physiological mechanisms (Herborn et al. 2010). The conspicuous courtship behaviour of male collared flycatchers, Ficedula albicollis, allows field assays to measure behavioural responses to ecologically relevant situations in natural conditions, without the capture of individuals (Garamszegi et al. 2008, 2009b). Previous studies using this system have shown that the within- and between-individual variation in three behaviours elicited in different contexts corroborates the behavioural syndrome concept: individuals display consistent behaviours over time (Garamszegi et al. 2006) and the correlation between these main components of behavioural syndromes varied between 0.37 and 0.77 (Garamszegi et al. 2009b). Hormone-mediated physiological mechanisms can also be traced using non-invasive approaches, as the levels of excreted CORT metabolites in the animals' droppings can be characterised via enzyme immunoassay (EIA). Such excreted metabolite levels are proven to be predictor of hormone concentrations in the blood in both mammals and birds (Palme et al. 2005).

The aim of this study was to test for the correlation between CORT levels and three components of behavioural syndromes (novelty avoidance, aggression and risk-taking) in a wild population of the collared flycatcher. We first assessed avoidance (i.e. behavioural response to a novelty situation), aggression (response to a social challenge) and risk-taking (response to a predator stimuli) within the breeding territory and then collected dropping samples from the same birds to quantify CORT metabolites. One should be careful with making interpretations for the causal mechanisms from such indirect estimates of hormone levels, because metabolite levels in the droppings reflect the mixture of circulating baselineand stress-induced levels over several hours before sampling (Goymann et al. 2002). Therefore, we tested for the correlation between behavioural and physiological traits by considering two possibilities. First, we predicted that if excreted CORT metabolite levels reflect baseline hormone concentration in the blood and if CORT levels have pleiotropic effects and mediate behavioural syndromes, all measured behavioural traits should covary with the measured hormone trait in the same direction, and these correlations should be of comparable magnitude with the strength of the relationship between behavioural traits. Specifically, high amounts of excreted CORT metabolites should be associated with higher avoidance, non-aggressive and risk-averse behaviours, while low metabolite levels should accompany lower avoidance, aggressive and risk-taking behaviours. It is also possible that not all behaviours are modulated by adreno-cortical hormones (i.e. CORT does not have pleitropic effects), thus only one or two behaviours will be related to CORT metabolite concentrations. Second, if the environmental challenges can have consequences for CORT metabolite levels (and not physiological status determines the behavioural responses), it should raise a correlation between the physiological stress response and behavioural reactions. Accordingly, we compared the concentration of CORT metabolites of males that were or were not subjected to the experimental challenges. If the expositions to a novel object, territorial intruder or a potential predator raise the level of stress hormones, we predict that individuals that were involved in the behavioural tests have higher concentration of CORT metabolites than individuals, which were not exposed to any of these stimuli.

Given the correlative nature of the study and the shortcomings associated with hormonal sampling, we present the detected relationships to extend our knowledge on hormone/behaviour relationships but not to challenge hypotheses about the causal mechanism.

\section{Materials and Methods}

\section{Study Species and General Methods}

Our study species, the collared flycatcher is a small migratory, socially monogamous (facultative polygyny may occur), hole-nesting passerine (Cramp \& Perrins 1994). After spring migration, males occupy territories and start singing and displaying to attract females. Paired females build nests alone, generally 
lay and incubate 6-7 eggs, while both sexes provide parental care at the nestling stage. We studied behaviour and physiology during the field season of 2007, 2009 and 2010 in a breeding plot at Pilis Field Station near Budapest $\left(47^{\circ} 43^{\prime} \mathrm{N}, 1^{\circ} 01^{\prime} \mathrm{E}\right)$, Hungary, that was established in 1981 (see Török \& Tóth 1988). First, we recorded the behaviour of unpaired males, and then, we captured them and took a dropping sample for subsequent hormone assay. We have chosen males for our purposes, because they show typical nest presentation behaviour and their elaborate courtship and territorial behaviour can be well characterised in different situations. In addition, during their display period, we were able to design protocols that allowed the measurement of behavioural traits without requiring the capture of individuals.

\section{Behavioural Traits}

Male behaviour was monitored during the most active morning singing period (usually between $6.00 \mathrm{am}$ and $12.00 \mathrm{pm})$, when males are intensively engaged in attracting females. To measure avoidance, we characterised the behaviour of resident males towards an experimentally changed breeding environment (altered nest box) relative to a familiar breeding environment (unaltered nest box). The alteration of the nest box appearance creates a situation, in which the activity of individuals in the presence of a novel object reflects exploration-avoidance sensu Réale et al. (2007) that we also followed for definitions. Note that based on these definitions, we systematically avoided adopting neophilia/neophobia terminologies in association with behaviours in response to a new situation, as used by some studies (Corey 1978; Greenberg 1990; Mettke-Hofmann et al. 2002). This is because from the evolutionary perspective, exploration-avoidance is the main subject to selection, and it includes reactions to any novelty (new habitat, new food and novel objects) by definition (Réale et al. 2007). On the other hand, neophilia or neophobia would deal with the mechanisms responsible for individual variation in behaviour (Greenberg \& Mettke-Hofmann 2001). We also note that in our previous publications (Garamszegi et al. 2008, 2009b), we labelled the avoidance scores at the altered breeding environment as exploration.

We first quantified the natural nest presentation (courtship) behaviour of males, which was stimulated by a decoy female placed on the top of the nest box (naturally, females often observe nest-presenting males from this position). Displaying males frequently approach and enter the nest box to call females from inside. These approaches involve excited flights around the nest box and repeated landings on top of the nest box and on the entrance hole. These behavioural patterns were characterised to describe the activity at the familiar breeding site during courtship (control activity). To estimate activity in an altered breeding situation (experimental activity), we modified the front of the nest box by attaching a white paper sheet below the entrance hole immediately after the control treatment and measured the same behavioural traits by using the same female stimuli. In both the control and experimental situations, the observer recorded courtship activity from a distance of ca. 25-30 m during a 5-min period. The focal behavioural trait was latency time (in sec) elapsed until the first landing of males on the entrance hole, measured from the appearance of the male on its territory (i.e. when the typical nest box presentation behaviour indicated that the male detected the female). Our previous study showed that this latency measure strongly correlated with other behavioural traits reflecting courtship activity (Garamszegi et al. 2009b). If a male did not land on the entrance hole during the observation period, it was considered to have 301 s latency (control situation: $N=4$, experimental situation: $\mathrm{N}=23$ ). From the two estimates of courtship latency, we expressed avoidance as the experimental activity relative to the control activity. This was simply the difference between the two variables after $\log _{10}$ transformation, which are the residuals of the $x=y$ regression line drawn between control and experimental activity. As shy animals may require longer time to accept the novel object and thus to land on the entrance hole, high values of residual courtship latency indicated higher avoidance behaviours in the altered breeding opportunity than lower values. Such avoidance score was normally distributed. We could obtain information on avoidance for $\mathrm{N}=51$ males, for which concentration of CORT metabolites in droppings was also available. There were additional 35 males with information on avoidance, for which no data on CORT metabolite levels could be gathered. However, avoidance score did not differ significantly between males of these two categories $\left(\mathrm{t}_{84}=0.641, \mathrm{p}=0.525\right)$, thus missing data on CORT metabolite levels should occur randomly with respect to the question of the study.

Following the avoidance tests, we quantified the aggressive behaviour of the resident males towards a territory intruder using a previously developed field assay (see Garamszegi et al. 2006 for more details). Briefly, we simulated natural territory intrusions by presenting a live male decoy in a small wire cage positioned 2-3 $\mathrm{m}$ from the nest box to the focal males. 
We measured the latency (in sec) between the detection of the stimulus male and the first attack, which is the event when the resident male touched the cage of the stimulus male, usually showing clear intention to fight. The detection of the stimulus bird was the moment when the focal male was first seen in the close proximity of the introduced cage (e.g. within 15 $-20 \mathrm{~m}$ ) with a high potential to detect the stimulus. Resident birds displayed typical behavioural signs in such a situation, for example, excited flights and display of vocalisations around the intruder, and we never observed them preening or scratching close to the decoy. Therefore, we assume that our ability to detect the male in his territory was not biased by its response to the presence of the intruder. Based on previous observations on the known variance in these behavioural traits (Garamszegi et al. 2006), if the resident male did not attack the decoy bird within 5 min, it was treated as non-aggressive, and it was assigned an attack latency score of $301 \mathrm{~s}(\mathrm{~N}=10)$. Attack latencies were treated as the inverse estimates of aggression, thus high values indicated less aggressive behaviour than small values. The latency to attack has been shown to be a good marker of the territorial behaviour in general, because it is repeatable across different trials and predicts the frequency and duration of subsequent attacks (Garamszegi et al. 2006). Aggression could be recorded for $\mathrm{N}=91$ males with $\mathrm{N}=56$ males also providing samples for CORT metabolite analysis. There was no statistical difference in aggression score between males with and without data on CORT metabolites $\left(\mathrm{t}_{89}=-0.220, \mathrm{p}=0.826\right)$.

Finally, risk-taking was assessed by estimating the distance at which an individual flees when a potential predator (human) was approaching (Blumstein et al. 2004; Blumstein 2006; Møller et al. 2008). After the assessment of first attack latency in the aggression test, the focal male was allowed to be engaged in territorial defence. When it had been located on the cage showing clear indication to fight, the observer moved, from a distance of c.a. 25-30 m, at a normal walking speed towards the resident male. When it took flight from the decoy because of the human approach, the observer stopped walking. Aggressive birds that focus heavily on the fight with the territorial intruder may only notice the approaching human at a close distance, thus they may appear irresponsive to the presence of a potential predator simply because they do not see them and not because they take more risk. To avoid this problem, the resident male was allowed to return fighting after the first flee, while the observer remained in the same position (i.e. the observer were ascertain that the focal bird is aware of the situation). If the bird returned to the decoy, the observer continued the approach, until the bird flew again. This was repeated as long as the focal male was re-engaged in the territorial dispute. The distance between the experimental cage and the observer, at which the male was last observed fighting was measured in number of steps (which approximately equals metres). Birds with short flight distance can be considered as risk takers, as they truly allow potential predators to approach until close proximity. We estimated flight distance when males interacted with others, thus motivation to fight may be a confounder. However, we previously found that flight distance during fighting correlates with the distance at which the same bird could be approached in another context, when singing (Garamszegi et al. 2008). Therefore, we assumed that flight distance is inversely related to risk-taking. We could record this estimate of risk-taking for $\mathrm{N}=89$ individuals. Among these birds, dropping samples could be obtained for $\mathrm{N}=54$ males. Missing data on CORT metabolites occurred randomly with respect to risk-taking $\left(\mathrm{t}_{87}=1.140, \mathrm{p}=0.257\right)$.

In 2009, after the first round of observations, we followed the courtship activity of the assayed birds based on their individual colour marks on the belly (we painted them with water-resistant pens upon capturing). If an individual was found singing on a territory few days later $(0-6 \mathrm{~d})$, we repeated the behavioural tests, and obtained additional information (including 1 -4 measurements per male) for avoidance, aggression and risk-taking as defined previously. Based on such multiple data, we could calculate the repeatability of the traits using mixed models (Nakagawa \& Schielzeth 2010). Within-individual variations compared with the total variation revealed repeatabilities (restricted maximum-likelihood estimates of repeatability from linear mixed effects model, avoidance score: $\mathrm{R}=0.309, \mathrm{CI}=$ 0.000/0.577, $p=0.069$; aggression score: $R=0.383$, $\mathrm{CI}=0.088-0.616, \quad \mathrm{p}=0.015$; risk-taking score: $\mathrm{R}=$ $0.608, \quad \mathrm{CI}=0.331 / 0.788, \mathrm{p}<0.001) \quad($ Table 1$)$ that were comparable with other behavioural traits (Bell et al. 2009).

Table 1: Restricted maximum-likelihood estimates of repeatability of the measured behavioral traits as obtained from linear mixed effects models

\begin{tabular}{lllr}
\hline & $\mathrm{R}$ & $95 \% \mathrm{Cl}$ (upper/lower) & \multicolumn{1}{c}{$\mathrm{p}$} \\
\hline Avoidance & 0.309 & $0.000 / 0.577$ & 0.069 \\
Aggression & 0.383 & $0.088 / 0.616$ & 0.015 \\
Risk taking & 0.608 & $0.331 / 0.788$ & $<0.001$ \\
\hline
\end{tabular}


We made an effort to use different stimulus birds across different tests, as the identity and attributes of presented males and females may be important (see Garamszegi et al. 2006; Hegyi et al. 2008). We exchanged stimulus birds, as they became available from our parallel capturing sessions at a distant breeding plot. Therefore, we assumed that the focal birds were unfamiliar with the stimulus birds. By using mixed-effect modelling with the identity of stimulus birds as random factor, we checked whether using the same decoy birds multiple times may have confounded the outcome of the study, but this was not the case.

Behavioural responses to the stimuli may be mediated by territory quality, because males may invest more in the defence and exploration of territories of superior quality than may do at lower-quality nest boxes. Hence, the relationship between the measured traits may not necessarily reflect behavioural syndromes but can be an artificial result arising from the non-standardised environment. In our previous studies, we carefully examined this possibility and showed that the behavioural correlations remain when we control for territory quality (Garamszegi et al. 2008, 2009b). Moreover, from the repeatability analyses of traits based on the multiple within-year observation of the same males, it was evident that behavioural scores were independent of whether the male retained or changed his territory during the course of sampling (avoidance score: $F_{1,35}<0.001$, p $=0.999$; aggression score: $\mathrm{F}_{1,38}=0.046, \mathrm{p}=0.832$; risk-taking score: $\left.F_{1,31}=0.148, p=0.703\right)$. Therefore, we assume that the correlation among the measured behaviours do reflect behavioural syndromes.

\section{Dropping Sampling and Hormonal Analyses}

After the behavioural tests, we captured resident males using a conventional nest box trap (traps were checked every $10 \mathrm{~min}$ to minimise stress). There were differences among males in the time needed to capture them, which lead to variation in the time delay between the beginning of the behavioural observation and sampling of droppings (mean $\pm \mathrm{SE}=2.44 \pm$ $0.15 \mathrm{~h})$. Therefore, a potential timing effect on estimated CORT metabolite levels was considered in the statistical analyses (Table 2). Captured birds were placed in a standard bird bag, the bottom of which was lined with a plastic bag to collect the droppings. Bags were checked after few minutes for fresh droppings (not more than $5 \mathrm{~min}$, which is the time needed for the capturer to reach the sampling station). If a sufficient amount of sample was found, we trans-
Table 2: The relationships between excreted corticosterone concentration in the droppings and some potentially confounding variables that may affect the relationship between behavioural and hormonal measurement in the collared flycatcher

\begin{tabular}{lllll}
\hline Predictor & $\mathrm{r}$ & $\mathrm{N}$ & $\mathrm{p}$ & $95 \% \mathrm{Cl}$ (lower/upper) \\
\hline Year & 0.051 & 58 & 0.702 & $-0.210 / 0.305$ \\
Date & 0.025 & 58 & 0.852 & $-0.235 / 0.281$ \\
$\begin{array}{l}\text { Time of scoring the } \\
\text { behaviour }\end{array}$ & 0.050 & 49 & 0.732 & $-0.234 / 0.327$ \\
$\begin{array}{l}\text { Time between scoring } \\
\text { avoidance and sampling } \\
\text { of droppings }\end{array}$ & -0.014 & 48 & 0.829 & $-0.295 / 0.268$ \\
$\begin{array}{l}\text { Age } \\
\text { Body mass }\end{array}$ & -0.077 & 57 & 0.557 & $-0.331 / 0.188$ \\
\hline
\end{tabular}

ferred it to an empty, dry plastic tube. As it has been demonstrated that in birds, CORT metabolites are excreted after a short time (Goymann et al. 2002), birds that had not produced any dropping within 5 min were excluded from the analysis to avoid that capture and handling stress would further elevate the stress-induced (CORT) levels. Therefore, CORT metabolite levels could not be estimated for all birds with information on behavioural traits. Collected samples were stored in a cool bag until reaching the laboratory, where they were kept frozen at $-20^{\circ} \mathrm{C}$ until extraction.

The concentrations of CORT metabolites were assessed via an EIA as described by Rettenbacher et al. (2004), a method that has already been validated for a closely related species, the pied flycatcher, F. hypoleuca (Lobato et al. 2008). Samples were extracted as described previously and aliquots of the supernatant were measured after dilution with assay buffer 1:10 in the respective assay. The quantification of immunoreactive CORT metabolites is described in detail in Rettenbacher et al. (2004).

We estimated the reliability of the CORT metabolite assay by calculating the repeatability of the estimated levels that were obtained from the analysis of different extracts of the same samples $(\mathrm{N}=17)$ and reanalyses of the same extracts $(\mathrm{N}=24)$. For the calculations, we used the methods for repeatability from Nakagawa \& Schielzeth (2010). Although faecal samples are not homogeneous, taking multiple extracts from different parts of the droppings resulted in more or less similar individual-specific values of CORT metabolite concentrations (restricted maximum-likelihood estimates of repeatability from linear mixed effects model: $\mathrm{R}=0.653$, 95\% CI range $=0.288-0.855, \mathrm{p}<0.001$ ). Still, in further analyses, we thoroughly mixed the droppings before extraction to avoid any bias. Performing repeated analyses on the same extract gave consis- 


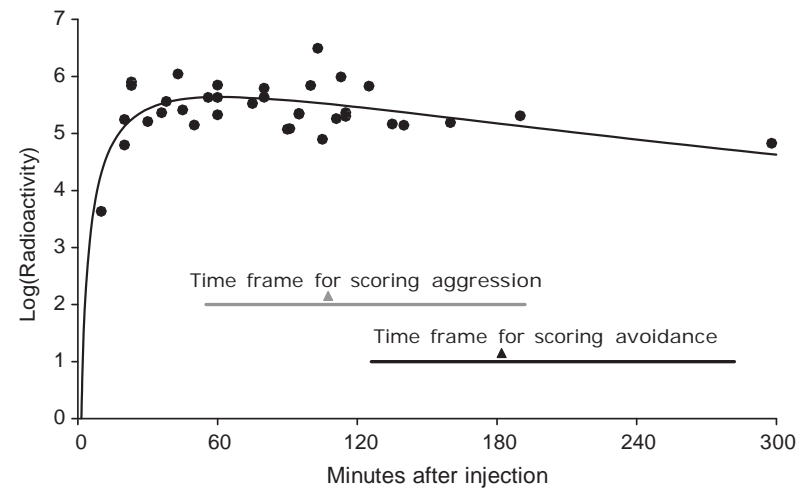

Fig. 1: The time course of the excretion of corticosterone metabolites following a hormone injection. Radioactively labelled corticosterone was injected into captive birds, and radioactivity was determined from subsequent droppings that were repeatedly collected after injection. The line is the regression line from the quadratic polynomial regression of $\log _{10}$-transformed data (see the corresponding statistical design in the main text). This time window was fitted to time frames of our behavioural observations. Grey and black bars represent the time elapsed between the given behavioural test and the collection of samples of droppings.

tent variation between individuals (correlation-based estimates of repeatability: $R=0.781, \quad 95 \% \quad$ CI range $=0.527-0.907, \mathrm{p}<0.001$; here we used the correlation method, because, unlike mentioned earlier, the reanalyses took place about a year later than the original analysis and so, owing to evaporation or degradation processes, the range of the absolute values of the metabolite concentrations are not comparable between analyses). Also when the same samples were reanalysed within a few days, estimates originating from the different analyses were highly repeatable (restricted maximum-likelihood estimates of repeatability from linear mixed effects model when statistically controlling for any plate, within plate position and day effects: $R=0.937,95 \%$ CI range $=0.790-$ 0.998, $\mathrm{p}<0.001$ ). Therefore, we can reasonably assume that despite variations caused by the relatively high inter- and intra-assay variance of the EIA the measured concentrations mirror biologically relevant individual-specific values. As CORT metabolite concentrations in the dropping samples were analysed blindly with respect to the behavioural data, any noise caused by measurement errors should only raise random noise and not systematic bias.

We also validated the used measurements via analytical and biological validation protocols, as recommended by Touma \& Palme (2005). In a first step, we performed a radiometabolism study to determine the excretion pattern of CORT, as well as HPLC immunogramms to characterise the excreted metabolites (Palme et al. 2005). As a second step, we assessed the biological validity of the method by testing whether an increase in excreted metabolites caused by enhanced adrenocortical activity could be detected. In total, 6 caged birds (both females and males, but sex effects were considered statistically) were administered radiolabelled ${ }^{3} \mathrm{H}$-corticosterone into the wing web. The hormones were dissolved in $0.9 \% \mathrm{NaCl}$ solution, so that the injected solvent contained $<5 \%$ $(\mathrm{v} / \mathrm{v})$ ethanol. The $\left[1,2,6,7-{ }^{3} \mathrm{H}(\mathrm{N})\right]$-corticosterone (2830.5 GBq/mmol) was obtained from New England Nuclear (Dreieich, Germany). The purity was previously checked via thin layer chromatography (Palme et al. 1997). After injection, we checked for fresh droppings every 15-20 min for a period of few hours resulting in 2-7 repeats per bird covering 10-298-min time interval. Radioactivity was determined as described by Rettenbacher et al. (2004) in each of the collected samples, and we drew a time plot to estimate the time course of excretion of the radiolabelled hormone metabolites (Fig. 1).

\section{Confounding Variables}

As variation in excreted CORT metabolite levels may not only depend on behavioural variables, we also assessed the effect of some potentially confounding factors that may interact with both hormone levels and behavioural traits. For example, CORT levels can vary in time, as it can follow a daily or a seasonal rhythm (Carere et al. 2003; Goymann et al. 2006; Quillfeldt et al. 2007; Li et al. 2008). Similarly, behaviour may also show temporal fluctuations (e.g. birds are more active in the morning). Therefore, we recorded the year, dates and exact time of behavioural observations to statistically control for their potential effect. Date was standardised between years using the difference (in days) from the median arrival date of the population in the given year. The date of observation (the date of behavioural tests) was used to reflect arrival date. We assumed that the date of observation is a good surrogate of real arrival date, because we monitor our breeding plots for newly arrived, displaying birds in a standard way. It is therefore likely that males used in this study were recorded just upon their arrival. Arrival order of males translates to differences in territory quality, as early arrival is generally known to lead to the occupation of prime breeding sites (Kokko 1999). Therefore, we used arrival date as a proxy variable for territory quality and took it into account as a potential confounder on our behavioural traits that were assessed in non-standard environment, as aggression, avoidance and risk-taking might be affected by territory quality (Garamszegi et al. 2009b). 
We also calculated the time interval between the behavioural observation and sampling to separate any effect owing to differences in time needed for sampling.

Age may also influence how individuals handle environmental challenges, as young and inexperienced individuals may be more likely to perceive a novel situation as a threat (Cavigelli \& McClintock 2003). Previous studies have repeatedly shown a relationship between age and CORT levels (Sockman \& Schwabl 2001; Angelier et al. 2006), but similar evidence has also been reported for explorative behaviour (Carere et al. 2005). Hence, we also collected data on male age. Based on the typical sub-adult plumage coloration of yearling males (brown remiges), we classified individuals as yearlings $(\mathrm{N}=12)$ or older birds ( $\mathrm{N}=45)$ (Svensson 1984).

Birds can exhibit varying stress response depending on their body mass (Schoech et al. 1997; Cockrem et al. 2006), whereas aggression and dominance status may also be influenced by body weight (Polo \& Bautista 2002; Jonart et al. 2007). Therefore, we also took measurements of body mass with a Pesola spring balance to the nearest $0.1 \mathrm{~g}$.

\section{The Effect of Behavioural Tests on Corticosterone Level}

To investigate the possibility that behavioural responses to the stimuli used in the experiment cause changes in CORT metabolite levels and not vice versa, in 2010, we also collected dropping samples for EIA assay from males that were not used in the behavioural tests $(\mathrm{N}=19)$. For these untested birds, we followed the same protocols as for the experimental birds (i.e. collected droppings after $5 \mathrm{~min}$ to avoid handling stress, etc.). Morphological characters, arrival dates and subsequent breeding success did not differ between the two groups of birds assayed in the same year (data not shown). We compared the metabolite concentrations of droppings produced by males with and without prior exposure to a novel object, territorial intruder or a potential predator. The null hypothesis for this comparison was that these groups did not differ, if our stimuli represented natural challenges that birds permanently underwent during the courtship period. Similarity in the measured CORT metabolite levels between the two groups of birds would imply that estimated concentrations reflect basal rather than stress-induced levels.

\section{Statistical Analyses}

Variables were checked for normality, and statistical transformations were applied if it was necessary to meet the normality assumption (concentrations of CORT metabolites, attack latency, flight distance, time estimates, radioactivity were $\log _{10}$-transformed). Given that transformations did not result in normal distribution for aggression owing to the relatively large number of 0 and 301 s latency measures, we applied nonparametric statistics for this variable.

To determine the strength and direction of the relationship between the behavioural and physiological traits, we estimated the Pearson's r correlation coefficients between them. The effect of the considered potentially confounding variables was investigated by using partial correlations, in which the variance caused by the unwanted factors could be accounted for. For example, by applying such an approach we tested whether the strength and direction of the relationship between CORT metabolite levels and behavioural traits were independent of the time delay between faecal sampling and behavioural observations. Initially, we constructed complex mixed models that included plate and column effects (i.e. to control for the physical position of the samples in the EIA in terms of plate and column number) as well as the identity of the decoy birds, but as the statistical control for these variables did not affect the results qualitatively, we present the outcome from simpler models relying on partial correlations without the consideration of random effects. Hormonal and radioactivity data originating from the repeated sampling of captive individuals were processed using a GLMM design, in which multiple entries from the same individual could be handled using random effect terms. As we expected that from an initial zero value, radioactivity would initially increase then decrease owing to excretion, we modelled the change of radioactivity as a second-order function of time elapsed from injection. The models contained sex as an additional independent variable (fixed factor) to deal with potential difference between male and female subjects. To compare CORT metabolite levels between groups of males with different history in behavioural tests, we used an unequal variance unpaired t-test (Ruxton 2006), because we had found that group variances were significantly different $\left(F_{1,36}=6.297, \mathrm{p}=0.017\right)$.

Because interpretations relying on significance levels are misleading owing to conceptual and philosophical reasons (see over 400 citations in http://welcome. warnercnr.colostate.edu/ anderson/thompson1.html), this paper follows interpretations based on the effect size theorem (Nakagawa 2004; Garamszegi 2006; Nakagawa \& Cuthill 2007; Garamszegi et al. 2009a). Accordingly, we focus on the magnitude of the biolog- 
ical relationships as reflected by effect size (such as Pearson's r sensu Cohen 1988) and on the precision by which these effects could be estimated from the current sample in terms of 95\% confidence intervals (CIs). We subsequently report 95\% CI ranges by presenting the lower and upper boundaries as lower limit/upper limit. As Cohen (1988) recommended, $r=0.1$ can be generally considered a small effect, $r=0.3$ a medium effect and $r=0.5$ a strong effect. Non-overlapping CIs that are narrow enough to allow distinction between these benchmarks of effect sizes imply high precision. For demonstrative purposes, we present $p$ values for each relationship. We avoid emphasising statistical significance, but bring attention to the biological importance of effects, while anticipating the uncertainty resulting from the data.

\section{Results}

Validation of the Measurement from Faecal Samples

We expressed the excretion of radioactive CORT metabolites in captive birds as the function of time elapsed from the injection. The corresponding GLMM analysis that controlled for individual effects revealed that radioactivity steeply increased until reaching a maximum at $60 \mathrm{~min}$ after the injection, then it showed a slowly declining tendency (time: $F_{1,26}=$ 3.492, $\quad p=0.073, \quad r=0.344, \quad C I=-0.033 / 0.636$; time*time: $F_{1,27}=40.075, \mathrm{p}<0.001, r=0.517, \mathrm{CI}=$ 0.572/0.893; Fig. 1). This was independent of whether the subject bird was a female or male, as adding sex and sex*time interaction as additional factors to the model does not suggest an important role for sex (sex: $\mathrm{F}_{1,24}=0.478, \mathrm{p}=0.496, \quad \mathrm{r}=0.140, \mathrm{CI}=-0.262 / 0.500$; time*sex: $F_{1,25}=2.148, \quad p=0.156, \quad r=0.287, \quad C I=$ -0.113/0.607).

We matched these time patterns with the course of our behavioural experiments (Fig. 1). We started our behavioural assays to score avoidance rate on average $182 \mathrm{~min} \quad(\mathrm{SE}=10.6 \mathrm{~min}, \quad$ range $=126-282 \mathrm{~min}$ ) before collecting dropping samples, while aggression was observed on average $107.5 \mathrm{~min}$ (SE $=8.2 \mathrm{~min}$, range $=55-192 \mathrm{~min}$ ) prior to the hormonal sampling (we did not estimate time frame for scoring risk-taking, as this observation was made immediately after the end of the assessment of aggression raising maximum 5-min difference). This time window implies that CORT metabolite concentrations measured in the droppings are likely to reflect the circulating levels of CORT during behavioural observations, because the time elapsed is sufficient for the metabolism and excretion of the hormone.
However, the time frame between behavioural manipulation and sampling of droppings might be long in some cases. In the related pied flycatcher, F. hypoleuca, a peak of CORT increase in the blood can be detected in the dropping c.a. $1.5 \mathrm{~h}$ after the effective peak (Lobato et al. 2008), which we often exceeded when sampling collared flycatchers after the behavioural tests. Hence, we cannot exclude the possibility that individuals that were difficult to catch were represented with CORT levels that correspond to different biological situation (after test) than individuals that were quickly captured (during test). Therefore, we further considered whether the time window between observation and sampling in some cases could affect the focal relationship.

\section{Confounding Variables}

The correlation matrix for the relationship between CORT metabolite concentrations in droppings and the considered confounding variables is given in Table 2 . Inspection of these effect sizes and the associated CIs suggests that these variables are unlikely to strongly influence levels of excreted CORT metabolites. However, considering the CIs of the estimates, we cannot exclude that body mass may play a partial role. Therefore, we decided to control for body mass in a multivariate approach. Moreover, we also considered further the potentially confounding effect of territory quality and the time interval between behavioural observations and sampling of droppings for the theoretical reasons detailed previously.

\section{Correlation between CORT and Behavioural Traits}

We failed to find a strong relationship between avoidance estimated as residual courtship latency and the concentration of CORT metabolites $(r=-0.017$, $\mathrm{N}=51, \mathrm{CI}=-0.291 / 0.260, \mathrm{p}=0.907$; Fig. 2a). Similarly, attack latency, our inverse approximate of aggression did not strongly forecast levels of excreted CORT metabolites $\left(r_{\mathrm{s}}=-0.076, \mathrm{~N}=56, \mathrm{CI}=-0.332 /\right.$ 0.191 , $\mathrm{p}=0.577$ ). Finally, there was no suggestion for flight distance, our inverse measure of risk-taking, being strongly associated with our estimate of the level of the stress hormone $(r=0.074, \quad N=54$, $\mathrm{CI}=-0.198 / 0.335, \mathrm{p}=0.600$; Fig. 2c).

Behavioural syndromes are manifested in the form of behavioural correlations. Accordingly, by using males with information on hormone levels, we found that behavioural traits measured after three different challenging situations were positively correlated with each other (avoidance score - aggression score: $r_{s}=$ 

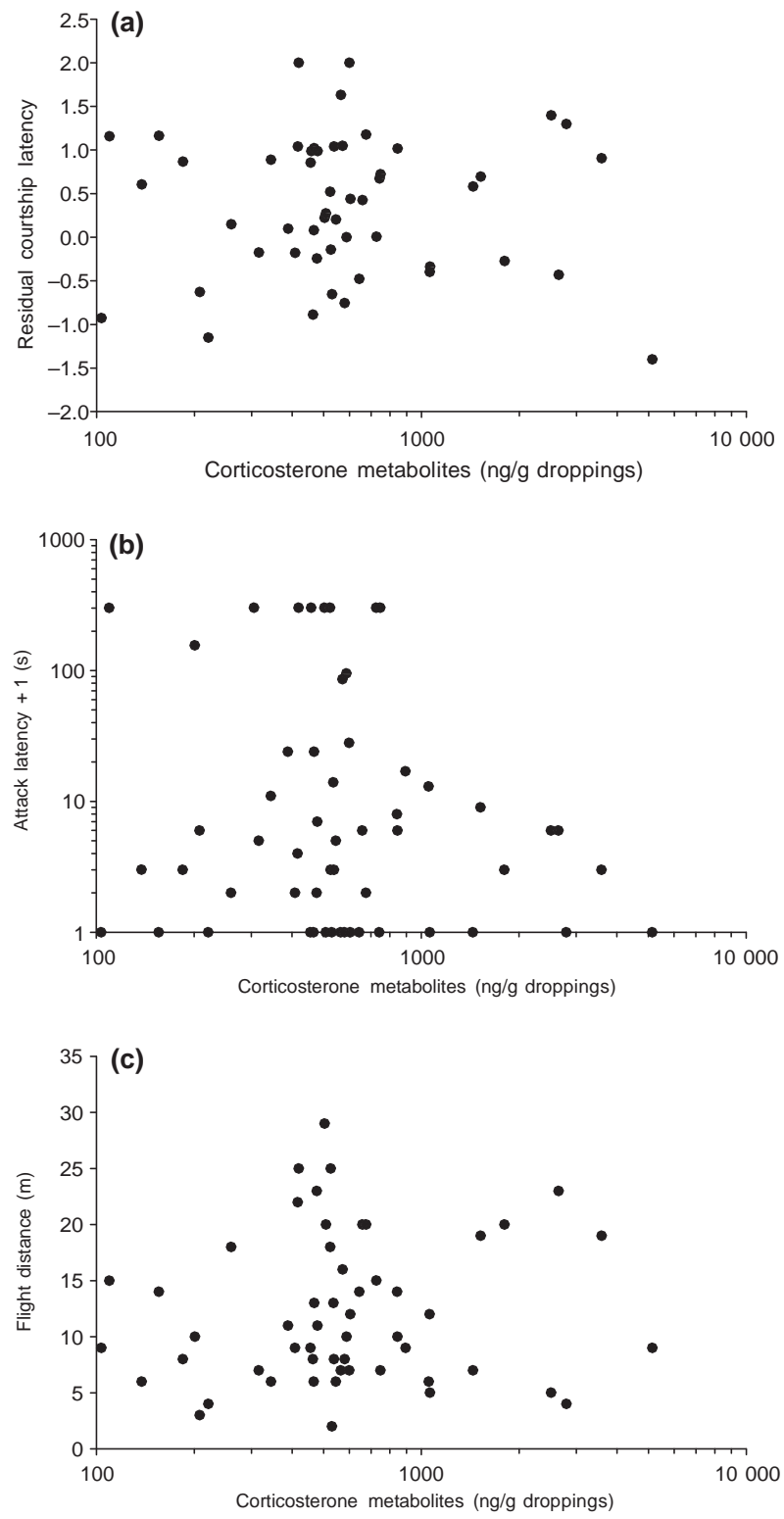

Fig. 2: The relationship between corticosterone metabolite levels in the droppings and components of correlated behaviours, (a) residual courtship latency (estimate of avoidance), (b) latency to attack an intruder bird (inverse estimate of aggression) and (c) flight distance (inverse estimate of risk-taking). Dots represent individual males.

$0.328, \quad \mathrm{~N}=50, \quad \mathrm{CI}=0.055 / 0.828, \quad \mathrm{p}=0.020 ;$ avoidance score - risk-taking score: $r=0.168, \mathrm{~N}=49, \mathrm{CI}=$ $-0.118 / 0.429, \mathrm{p}=0.247$; aggression score - risk-taking score: $r_{\mathrm{s}}=0.280, \mathrm{~N}=53, \mathrm{CI}=0.011 / 0.512, \mathrm{p}=0.042$; see also Garamszegi et al. 2009b). These results show that our sample sizes have the potential to detect effect sizes that reveal biologically important patterns. Therefore, it seems that the correlation between different behavioural traits is stronger than the correlation between CORT metabolite levels and behaviours.

We controlled for the effects of body mass by estimating partial correlations between behaviours and physiology while holding body size constant. Such correlations showed that previous mean effect size estimations were not altered qualitatively (avoidance score: $\mathrm{r}=-0.045, \mathrm{~N}=50, \mathrm{CI}=-0.319 / 0.236, \mathrm{p}=$ 0.757, aggression score: $r_{s}=-0.055, N=55, \quad C I=$ $-0.316 / 0.213, p=0.690$, risk-taking score: $r=0.021$, $\mathrm{N}=53, \quad \mathrm{CI}=-0.251 / 0.290, \quad \mathrm{p}=0.884) . \quad$ Similarly, effect sizes for the focal relationships between behaviours and CORT metabolite levels were not affected considerably when the time interval between behavioural observations and sampling of dropping were held constant (avoidance score: $r=0.019$, $\mathrm{N}=43, \quad \mathrm{CI}=-0.283 / 0.318, \quad \mathrm{p}=0.906, \quad$ aggression score: $r_{\mathrm{s}}=-0.073, \mathrm{~N}=47, \mathrm{CI}=-0.353 / 0.219, \mathrm{p}=$ 0.624 , risk-taking score: $r=0.083, \quad \mathrm{~N}=45, \quad \mathrm{CI}=$ $-0.216 / 0.368, p=0.594$ ). Finally, we also controlled for territory quality by using arrival date as predictor, but such a control is also confirmed our previous findings concerning the strength of relationship between physiology and behaviour (avoidance score: $\mathrm{r}=-0.037, \mathrm{~N}=51, \quad \mathrm{CI}=-0.309 / 0.241, \mathrm{p}=0.800$, aggression score: $r_{\mathrm{s}}=-0.102, \mathrm{~N}=56, \mathrm{CI}=-0.355$ / $0.165, \mathrm{p}=0.455$, risk-taking score: $r=0.066, \mathrm{~N}=54$, $\mathrm{CI}=-0.205 / 0.328, \mathrm{p}=0.638)$.

\section{The Effect of Behavioural Tests on CORT Level}

Metabolite levels of birds that were used in the behavioural tests in 2010 did not differ considerably from birds that were not subject to the behavioural tests in the same year (tested birds: mean \pm $\mathrm{SE}=508.6 \pm 44.54 \mathrm{ng} / \mathrm{g}$ droppings; non-tested birds: mean $\pm \mathrm{SE}=770.1 \quad \pm 134.9 \mathrm{ng} / \mathrm{g}$ droppings; effect size for the difference: $r=-0.204, N=38, C I=$ $-0.492 / 0.123, \mathrm{p}=0.220$ ). To exclude the possibility that the peak of CORT response remained undetected in birds that were sampled relatively late after the behavioural tests, we calculated mean CORT metabolite levels across birds for which the sampling of dropping occurred within $90 \mathrm{~min}$ after assessing behaviour (all years combined, mean $\pm \mathrm{SE}=591.3$ $\pm 42.19 \mathrm{ng} / \mathrm{g}$ droppings, $\mathrm{N}=11$ ). However, the mean CORT metabolite level in untested birds was not distinguishable statistically from this hypothetical mean (effect size for the difference: $r=-0.298, N=19$, $\mathrm{CI}=-0.663 / 0.181, \mathrm{p}=0.202$ ). In fact, the detected tendencies (the means are higher in non-tested than in tested birds) imply that we can virtually exclude the possibility that the exposition to novel object, ter- 
ritorial intruder or potential predator causes an increase in CORT metabolite levels compared with the levels of birds that received only natural stimuli during their courtship behaviour.

\section{Discussion}

The main finding of our study was that none of the investigated behavioural traits that form behavioural syndromes correlated strongly with levels of excreted CORT metabolites in territorial collared flycatcher males. These results suggest that increased or decreased rates of avoidance, intrasexual aggression and risk-taking are not associated with considerable physiological stress. Although we worked with larger sample sizes than other studies investigating the hormonal background of similar behaviours (e.g. Carere et al. 2003; Kralj-Fiser et al. 2007; Schoech et al. 2009), there was a noticeable uncertainty around the estimates of the effect sizes, as indicated by the broad CIs range. Therefore, instead of stressing that there is no effect, we emphasise that the relationship between CORT levels and behaviour, if it exists at all, is likely to be of small magnitude.

Our correlative findings do not lend strong support to the hypothesis that correlated behaviours are mediated by the pleiotropic effect of CORT. This hypothesis would predict that the expression of each behavioural trait corresponding to different ecologically challenging situations shows a systematic relationship with hormone excretion. Here, we found correlations of medium magnitude between residual courtship latency and attack latency (reflecting avoidance and inversely reflecting aggression, respectively) and between attack latency and flight distance (both inversely reflecting aggression and risk-taking, respectively), which imply a role for behavioural syndromes (see also Garamszegi et al. 2009b). However, levels of CORT metabolites in droppings did not relate to behavioural traits with comparable extent. The analysis in relation to aggression score provided a confidence interval around the estimated effect size that deviated slightly from zero effect. However, even if it would represent a biologically relevant association, it would mean that variations in hormone concentration could affect some of the variation in one behavioural trait, but without imposing changes in the same direction on other components of the behavioural syndrome contrary to the expectations from pleitropic effects.

Moreover, our results do not seem to corroborate the opposite causal scenario, in which the environmental stimuli or the solicited behaviour affect stress physiology. This mechanism to be at work would not predict CORT to be equally related to all components of behavioural syndromes. Only those behaviours that involve threat or stress to the individual are expected to show a relationship with the hormone. We performed an experiment, in which we compared CORT metabolite levels of birds that did or did not face challenges in the form of our behavioural tests. This part of the study reveals that particular stimuli and the elicited behaviours are unlikely to have consequence for CORT secretion. If particular behaviours involve different threats to the individual or differences in environmental challenge experienced, we should have detected different stress responses reflected by different CORT levels, which was not the case. Therefore, our results indicate that CORT metabolite levels of the tested birds are similar of those birds that only undergo natural stimuli from their environment during their courtship behaviour. Moreover, comparing natural excretion levels to that of captive birds, we found that the range of CORT metabolite levels measured after the behavioural tests (mean $\pm \mathrm{SE}=807.4 \pm 117.8 \mathrm{ng} / \mathrm{g}$ dropping) were considerably lower than levels that could be measured in birds that spent at least half an hour in captivity (mean $\pm \mathrm{SE}=1435.4 \pm 250.2 \mathrm{ng} / \mathrm{g}$ dropping, B. Rosivall, unpublished data). Therefore, we infer that the metabolite levels we found during courtship displays correspond to hormone levels that are confounded by lower stress response.

Our inability to find a considerable relationship between CORT and behaviour can be explained by at least three reasons. First, the weak relationships may imply that the measurement of behavioural and physiological variables was loaded with considerable measurement error that could have masked modest functional relationships in a correlative study. Behaviours are flexible traits and often show certain variation within individuals that generally leads to low to immediate repeatability (Bell et al. 2009). This was also the case in the current study (Table 1), but despite this, our sample was appropriate to detect biologically meaningful inter-individual correlations between the measured behavioural traits. Furthermore, other studies that relied on comparable sample size and tested for the relationship between CORT and different behavioural traits such as aggression and predation risk did find a correlation in the lab (Sorenson et al. 1997; Canoine et al. 2002; Landys et al. 2007).

Some methodological limitations should also be considered when interpreting the absence of strong correlations between behaviour and stress physiology based on faecal sampling of excreted metabolites, 
even if they provide reliable estimates. Faecal samples give an integrated estimate of circulating CORT levels over a certain time period that potentially include both baseline and stress responses (Goymann et al. 2002; Palme et al. 2005). Hence, the potential mixture of baseline and stress levels in the droppings may have prevented us to find strong correlations. Although we considered the potentially confounding effects of stress responses by controlling statistically for the time delay between the behavioural tests and faecal sampling and by comparing CORT metabolite levels between assayed and non-assayed birds, we cannot fully exclude the possibility that both the baseline and stress responses were mixed in our samples. Moreover, given that for practical reasons we performed three behavioural assays and then collected a single sample to estimate the level of CORT metabolites, our experimental design did not allow determining the relationships separately and independently for each behavioural trait. If stress response of a particular behaviour is generally stronger than that of other behaviours, the physiological consequences of different behaviours remain blurred in the combined sample. Consequently, our results should be interpreted with some caution.

The second reason behind the weak effects we found may be that there is no biological link between CORT and behaviour, as the measured traits may not necessarily belong to stress-related behaviours and may not be controlled by stress hormones. Moreover, we cannot exclude the possibility that CORT is not involved in the concerted organisation of behaviours, but other traits, if any, are responsible for pleiotropy. For example, behavioural syndromes may be modulated by neurotransmitter-associated genes (Fidler et al. 2007) or another hormone such as testosterone (van Oers et al. 2011). Moreover, it also remains plausible that no pleiotropic factors should be sought to explain the existence of correlated behaviours. If different behavioural phenotypes are beneficial in different situations (e.g. bold behaviours like aggression in social contexts, but shy behaviours in the presence of predator) there will not be strong selection for behavioural syndromes and evolution will decouple the physiological link between behaviours (Wilson 1998; Bell 2005; Dingemanse et al. 2007).

The third explanation for our negative findings stands on a mechanism in which individuals can control their stress levels via escape behaviours. If animals have an option to react to an environmental or social challenge by both behavioural and physiological means, it is not necessary that all stress factors will actually affect CORT levels (Cockrem \& Silverin 2002a; Goymann \& Wingfield 2004). Hormonal reactions may only be elicited in situations in which individuals have no behavioural control over the situation, for example, by escaping. Therefore, it is also plausible that we failed to detect strong relationships between behaviour and CORT metabolite levels, because the tested males had a choice to make their own decisions to approach the entrance hole, intruder or to escape from the human observer without the need of mobilising the physiological stress machinery. Hence, subject birds may have successfully adjusted their behaviour before any stress response would have initiated the CORT system.

To improve our understanding about the functional relationship between hormones and behaviour, future studies should discriminate experimentally between stress-induced and baseline CORT levels. For a better comprehension of the physiological regulation of behavioural syndromes, additional field studies are required, in which different behaviours are investigated in parallel as a cause or consequence of stress physiology.

\section{Acknowledgements}

During this study, LZG received a postdoctoral fellowship from the FWO Flanders (Belgium) and subsequently a research grant from the CSIC (Spain). BR was supported by a postdoctoral fellowship from the Hungarian Scientific Research Fund. G. Hegyi assisted in the capturing protocols. The fieldwork was supported by the Hungarian Scientific Research Fund (grants: T049678, K75618, F68295 and PD75481) and by the 'Plan Nacional' programme of the Spanish government (grant: CGL2009-10652). The financial support of the Austrian-Hungarian Bilateral Grant (NKTH-ÖAD, Project HU 12/2008) is gratefully acknowledged. GM was supported by the TÁMOP4.2.1./B-09/1-KMR-2010-0005 grant. We thank Amanda Aichinger and Wanda Kawinek for laboratory assistance. We are very grateful to the Pilis Park Forestry.

\section{Literature Cited}

Angelier, F., Shaffer, S. A., Weimerskirch, H. \& Chastel, O. 2006: Effect of age, breeding experience and senescence on corticosterone and prolactin levels in a longlived seabird: the wandering albatross. Gen. Comp. Endocrinol. 149, 1-9.

Arnold, S. J. 1992: Constraints on phenotypic evolution. Am. Nat. 140, S85-S107. 
Avitsur, R., Stark, J. L. \& Sheridan, J. F. 2001: Social stress induces glucocorticoid resistance in subordinate animals. Horm. Behav. 39, 247-257.

Bell, A. M. 2005: Behavioural differences between individuals and two populations of stickleback (Gasterosteus aculeatus). J. Evol. Biol. 18, 464-473.

Bell, A. M., Hankison, S. J. \& Laskowski, K. L. 2009: The repeatability of behaviour: a meta-analysis. Anim. Behav. 77, 771-783.

Belthoff, J. R. \& Dufty, A. M. 1998: Corticosterone, body condition and locomotor activity: a model for dispersal in screech-owls. Anim. Behav. 55, 405-415.

Blumstein, D. T. 2006: Developing an evolutionary ecology of fear: how life history and natural history traits affect disturbance tolerance in birds. Anim. Behav. 71, 389-399.

Blumstein, D. T., Ferná ndez-Juricic, E., LeDee, O., Larsen, E., Rodriguez-Prieto, I. \& Zugmeyer, C. 2004: Avian risk assessment: effects of perching height and detectability. Ethology 110, 273-285.

Canoine, V., Hayden, T. J., Rowe, K. \& Goymann, W. 2002: The stress response of European stonechats depends on the type of stressor. Behaviour 139, 13031311.

Carere, C., Groothuis, T. G. G., Mostl, E., Daan, S. \& Koolhaas, J. M. 2003: Fecal corticosteroids in a territorial bird selected for different personalities: daily rhythm and the response to social stress. Horm. Behav. 43, 540 -548 .

Carere, C., Drent, P. J., Privitera, L., Koolhaas, J. M. \& Groothuis, T. G. G. 2005: Personalities in great tits, Parus major: stability and consistency. Anim. Behav. 70, 795 -805 .

Carere, C., Caramaschi, D. \& Fawcett, T. W. 2010: Covariation between personalities and individual differences in coping with stress: converging evidence and hypotheses. Curr. Zool. 56, 728-740.

Cavigelli, S. A. \& McClintock, M. K. 2003: Fear of novelty in infant rats predicts adult corticosterone dynamics and an early death. Proc. Nat. Acad. Sci. U.S.A. 100, 16131 $-16136$

Cockrem, J. F. 2007: Stress, corticosterone responses and avian personalities. J. Ornithol. 148, S169—S178.

Cockrem, J. F. \& Silverin, B. 2002a: Sight of a predator can stimulate a corticosterone response in the Great tit (Parus major). Gen. Comp. Endocrinol. 125, 248-255.

Cockrem, J. F. \& Silverin, B. 2002b: Variation within and between birds in corticosterone responses of great tits (Parus major). Gen. Comp. Endocrinol. 125, 197-206.

Cockrem, J. F., Potter, M. A. \& Candy, E. J. 2006: Corticosterone in relation to body mass in Adelie penguins (Pygoscelis adeliae) affected by unusual sea ice conditions at Ross Island, Antarctica. Gen. Comp. Endocrinol. 149, 244-252.
Cohen, J. 1988: Statistical Power Analysis for the Behavioural Sciences. Lawrence Erlbaum Associates, Hillsdale.

Corey, D. T. 1978: The determinants of exploration and neophobia. Neurosci. Biobehav. Rev. 2, 235-253.

Cramp, S. \& Perrins, C. M. 1994: The Birds of the Western Palearctic. Oxford Univ press, Oxford.

Dingemanse, N. J., Wright, J., Kazem, A. J. N., Thomas, D. K., Hickling, R. \& Dawnay, N. 2007: Behavioural syndromes differ predictably between 12 populations of three-spined stickleback. J. Anim. Ecol. 76, 11281138.

Fidler, A. E., van Oers, K., Drent, P. J., Kuhn, S., Mueller, J. C. \& Kempenaers, B. 2007: Drd4 gene polymorphisms are associated with personality variation in a passerine bird. Proc. R. Soc. B-Biol. Sci. 274, 1685-1691.

Garamszegi, L. Z. 2006: Comparing effect sizes across variables: generalization without the need for Bonferroni correction. Behav. Ecol. 17, 682-687.

Garamszegi, L. Z., Rosivall, B., Hegyi, G., Szöllõsi, E., Törö k, J. \& Eens, M. 2006: Determinants of male territorial behavior in a Hungarian collared flycatcher population: plumage traits of residents and challengers. Behav. Ecol. Sociobiol. 60, 663-671.

Garamszegi, L. Z., Eens, M. \& Török, J. 2008: Birds reveal their personality when singing. PLoS One 3, e2647.

Garamszegi, L. Z., Calhim, S., Dochtermann, N., Hegyi, G., Hurd, P. L., Jørgensen, C., Kutsukake, N., Lajeunesse, M. J., Pollard, K. A., Schielzeth, H., Symonds, M. R. E. \& Nakagawa, S. 2009a: Changing philosophies and tools for statistical inferences in behavioral ecology. Behav. Ecol. 20, 1363-1375.

Garamszegi, L. Z., Eens, M. \& Török, J. 2009b: Behavioural syndromes and trappability in free-living collared flycatchers, Ficedula albicollis. Anim. Behav. 77, 803-812.

Gosling, S. D. 2001: From mice to men: what can we learn about personality from animal research? Psychol. Bull. 127, 45-86.

Goymann, W. \& Wingfield, J. C. 2004: Allostatic load, social status, and stress hormones - the costs of social status matter. Anim. Behav. 67, 591-602.

Goymann, W., Möstl, E. \& Gwinner, E. 2002: Noninvasive methods to measure androgen metabolites in excrements of European stonechats, Saxicola torquata rubicola. Gen. Comp. Endocrinol. 129, 80-87.

Goymann, W., Geue, D., Schwabl, I., Flinks, H., Schmidl, D., Schwabl, H. \& Gwinner, E. 2006: Testosterone and corticosterone during the breeding cycle of equatorial and European stonechats (Saxicola torquata axillaris and S. t. rubicola). Horm. Behav. 50, 779—785.

Greenberg, R. 1990: Feeding neophobia and ecological plasticity: a test of the hypothesis with captive sparrows. Anim. Behav. 39, 375-379. 
Greenberg, R. \& Mettke-Hofmann, C. 2001: Ecological aspects of neophobia and neophilia in birds. Curr. Ornithol. 16, 119-178.

Groothuis, T. G. G. \& Carere, C. 2005: Avian personalities: characterization and epigenesis. Neurosci. Biobehav. Rev. 29, 137-150.

Hegyi, G., Garamszegi, L. Z., Eens, M. \& Török, J. 2008: Female ornamentation and territorial conflicts in collared flycatchers (Ficedula albicollis). Naturwissenschaften 95, 993-996.

Herborn, K. A., Macleod, R., Miles, W. T. S., Schofield, A. N. B., Alexander, L. \& Arnold, K. E. 2010: Personality in captivity reflects personality in the wild. Anim. Behav. 79, 835-843.

Herczeg, G. \& Garamszegi, L. Z. 2012: Individual deviation from behavioural correlations: a simple approach to study the evolution of behavioural syndromes. Behav. Ecol. Sociobiol. 66, 161-169.

Jonart, L. M., Hill, G. E. \& Badyaev, A. V. 2007: Fighting ability and motivation: determinants of dominance and contest strategies in females of a passerine bird. Anim. Behav. 74, 1675-1681.

Jones, R. B., Blokhuis, H. J. \& Beuving, G. 1995: Openfield and tonic immobility responses in domestic chicks of 2 genetic lines differing in their propensity to feather peck. Br. Poult. Sci. 36, 525-530.

Ketterson, E. D. \& Nolan, V. J. 1999: Adaptation, exaptation, and constraint: a hormonal perspective. Am. Nat. 154, S4-S25.

Kokko, H. 1999: Competition for early arrival in migratory birds. J. Anim. Ecol. 68, 940-950.

Koolhaas, J. M., Meerlo, P., DeBoer, S. F., Strubbe, J. H. \& Bohus, B. 1997: The temporal dynamics of the stress response. Neurosci. Biobehav. Rev. 21, 775-782.

Koolhaas, J. M., Korte, S. M., De Boer, S. F., Van Der Vegt, B. J., Van Reenen, C. G., Hopster, H., De Jong, I. C., Ruis, M. A. W. \& Blokhuis, H. J. 1999: Coping styles in animals: current status in behavior and stressphysiology. Neurosci. Biobehav. Rev. 23, 925-935.

Koolhaas, J. M., de Boer, S. F., Buwalda, B., van der Vegt, B. J., Carere, C. \& Groothuis, A. G. G. 2001: How and why coping systems vary among individuals. In: Coping with Challenge: Welfare in Animals Including Humans (Broom, D. M., ed). Dahlem Univ Press, Dahlem, pp. 197-209.

Korte, S. M., Beuving, G., Ruesink, W. \& Blokhuis, H. J. 1997: Plasma catecholamine and corticosterone levels during manual restraint in chicks from a high and low feather pecking line of laying hens. Physiol. Behav. 62, 437-441.

Kralj-Fiser, S., Scheiber, I. B. R., Blejec, A., Moestl, E. \& Kotrschal, K. 2007: Individualities in a flock of freeroaming greylag geese: behavioral and physiological consistency over time and across situations. Horm. Behav. 51, 239-248.

Kralj-Fiser, S., Weiss, B. M. \& Kotrschal, K. 2010: Behavioural and physiological correlates of personality in greylag geese (Anser anser). J. Ethol. 28, 363-370.

Lande, R. 1992: Neutral theory of quantitative genetic variance in an island model with local extinction and colonization. Evolution 46, 381-389.

Landys, M. M., Goymann, W., Raess, M. \& Slagsvold, T. 2007: Hormonal responses to male-male social challenge in the blue tit Cyanistes caeruleus: singlebroodedness as an explanatory variable. Physiol. Biochem. Zool. 80, 228-240.

Lendvai, A. Z., Bokony, V. \& Chastel, O. 2011: Coping with novelty and stress in free-living house sparrows. J. Exp. Biol. 214, 821-828.

Li, D. M., Wang, G., Wingfield, J. C., Zhang, Z., Ding, C. Q. \& Lei, F. M. 2008: Seasonal changes in adrenocortical responses to acute stress in Eurasian tree sparrow (Passer montanus) on the Tibetan Plateau: comparison with house sparrow (P. domesticus) in North America and with the migratory P. domesticus in Qinghai Province. Gen. Comp. Endocrinol. 158, 47-53.

Lobato, E., Merino, S., Moreno, J., Morales, J., Tomas, G., La Puente, J. M. D., Osorno, J. L., Kuchar, A. \& Mostl, E. 2008: Corticosterone metabolites in blue tit and pied flycatcher droppings. Horm. Behav. 53, 295-300.

Martins, T. L., Roberts, M. L., Giblin, I., Huxham, R. \& Evans, M. R. 2007: Speed of exploration and risk-taking behavior are linked to corticosterone titres in zebra finches. Horm. Behav. 52, 445-453.

Mateos, C. 2005: The subordination stress paradigm and the relation between testosterone and corticosterone in male ring-necked pheasants. Anim. Behav. 69, 249255.

Mettke-Hofmann, C., Winkler, H. \& Leisler, B. 2002: The significance of ecological factors for exploration and neophobia in parrots. Ethology 108, 249-272.

Mettke-Hofmann, C., Rowe, K. C., Hayden, T. J. \& Canoine, V. 2006: Effects of experience and object complexity on exploration in garden warblers (Sylvia borin). J. Zool. 268, 405-413.

Møller, A. P., Nielsen, J. T. \& Garamszegi, L. Z. 2008: Risk taking by singing males. Behav. Ecol. 19, 41-53.

Nakagawa, S. 2004: A farewell to Bonferroni: the problems of low statistical power and publication bias. Behav. Ecol. 15, 1044-1045.

Nakagawa, S. \& Cuthill, I. C. 2007: Effect size, confidence interval and statistical significance: a practical guide for biologists. Biol. Rev. 82, 591-605.

Nakagawa, S. \& Schielzeth, H. 2010: Repeatability for Gaussian and non-Gaussian data: a practical guide for biologists. Biol. Rev. 85, 935-956. 
Odeh, F. M., Cadd, G. G. \& Satterlee, D. G. 2003: Genetic characterization of stress responsiveness in Japanese quail. 2. Analyses of maternal effects, additive sex linkage effects, heterosis, and heritability by diallel crosses. Poul. Sci. 82, 31-35.

van Oers, K., Buchanan, K. L., Thomas, T. E. \& Drent, P. J. 2011: Correlated response to selection of testosterone levels and immunocompetence in lines selected for avian personality. Anim. Behav. 81, 1055-1061.

Orchinik, M., Gasser, P. \& Breuner, C. 2002: Rapid corticosteroid actions on behavior. In: Hormones, Brain and Behavior, (Pfaff, D. W., Arnold, A. P., Etgen, A. M., Fahrbach, S. E. \& Rubin, R. T., eds). Academic Press, Amsterdam, pp. 567-600.

Palme, R., Mostl, E., Brem, G., Schellander, K. \& Bamberg, E. 1997: Faecal metabolites of infused C-14-progesterone in domestic livestock. Reprod. Domest. Anim. 32, 199-206.

Palme, R., Rettenbacher, S., Touma, C., El Bahr, S. M. \& Mostl, E. 2005: Stress hormones in mammals and birds: comparative aspects regarding metabolism, excretion, and noninvasive measurement in fecal samples. Ann. N. Y. Acad. Sci. 1040, 162-171.

Polo, V. \& Bautista, L. M. 2002: Daily body mass regulation in dominance-structured coal tit (Parus ater) flocks in response to variable food access: a laboratory study. Behav. Ecol. 13, 696-704.

Quillfeldt, P., Poisbleau, M., Chastel, O. \& Masello, J. F. 2007: Corticosterone in thin-billed prion Pachyptila belcheri chicks: diel rhythm, timing of fledging and nutritional stress. Naturwissenschaften 94, 919-925.

Réale, D., Reader, S. M., Sol, D., McDougall, P. T. \& Dingemanse, N. J. 2007: Integrating animal temperament within ecology and evolution. Biol. Rev. 82, 291-318.

Rettenbacher, S., Mostl, E., Hackl, R., Ghareeb, K. \& Palme, R. 2004: Measurement of corticosterone metab- olites in chicken droppings. Br. Poult. Sci. 45, 704-711.

Ruxton, G. D. 2006: The unequal variance t-test is an underused alternative to Student's t-test and the Mann-Whitney U test. Behav. Ecol. 17, 688-690.

Saldanha, C. J., Schlinger, B. A. \& Clayton, N. S. 2000: Rapid effects of corticosterone on cache recovery in mountain chickadees (Parus gambeli). Horm. Behav. 37, 109-115.

Sapolsky, R. M. 1994: Individual-differences and the stress-response. Sem. Neurosci. 6, 261-269.

Satterlee, D. G. \& Johnson, W. A. 1988: Selection of japanese quail for contrasting blood corticosterone response to immobilization. Poult. Sci. 67, 25-32.

Schoech, S. J., Mumme, R. L. \& Wingfield, J. C. 1997: Corticosterone, reproductive status, and body mass in a cooperative breeder, the Florida scrub-jay (Aphelocoma coerulescens). Physiol. Zool. 70, 68-73.

Schoech, S. J., Rensel, M. A., Bridge, E. S., Boughton, R. K. \& Wilcoxen, T. E. 2009: Environment, glucocorticoids, and the timing of reproduction. Gen. Comp.

Endocrinol. 163, 201-207.

Sih, A., Bell, A. \& Johnson, J. C. 2004a: Behavioral syndromes: an ecological and evolutionary overview. Trends Ecol. Evol. 19, 372-378.

Sih, A., Bell, A. M., Johnson, J. C. \& Ziemba, R. E. 2004b: Behavioral syndromes: an integrative overview. Q. Rev. Biol. 79, 241-277.

Silverin, B. 1997: The stress response and autumn dispersal behaviour in willow tits. Anim. Behav. 53, 451459.

Sockman, K. W. \& Schwabl, H. 2001: Plasma corticosterone in nestling American kestrels: effects of age, handling stress, yolk androgens, and body condition. Gen. Comp. Endocrinol. 122, 205-212.

Sorenson, L. G., Nolan, P. M., Brown, A. M., Derrickson, S. R. \& Monfort, S. L. 1997: Hormonal dynamics during mate choice in the northern pintail: a test of the 'challenge' hypothesis. Anim. Behav. 54, 1117-1133.

Stamps, J. A. 1991: Why evolutionary issues are reviving interest in proximate behavioral mechanisms. Am. Zool 31, 338-348.

Stö we, M., Rosivall, B., Drent, P. J. \& Mostl, E. 2010: Selection for fast and slow exploration affects baseline and stress-induced corticosterone excretion in Great tit nestlings, Parus major. Horm. Behav. 58, 864-871.

Svensson, L. 1984: Identification Guide to European Passerines. British Trust for Ornithology, Stockholm.

Török, J. \& Tóth, L. 1988: Density dependence in reproduction in the collared flycatcher (Ficedula albicollis) at high populatinn levels. J. Anim. Ecol. 57, 251-258.

Touma, C. \& Palme, R. 2005: Measuring fecal glucocortoids in mammals and birds: the importance of validation. Ann. N. Y. Acad. Sci. 1064, 54-74.

Wilson, D. S. 1998: Adaptive individual differences within single populations. Philos. Trans. R. Soc. Lond. B-Biol. Sci. 353, 199-205.

Wingfield, J. C. \& Romero, L. M. 2000: Adrenocortical responses to stress and their modulation in free-living vertebrates. In: Coping with the Environment (Mc Ewen, B., ed). Oxford Univ Press, New York, pp. 211-234.

Wolf, M., van Doorn, G. S. \& Weissing, F. J. 2008: Evolutionary emergence of responsive and unresponsive personalities. Proc. Nat. Acad. Sci. U.S.A. 41, 15825-15830. 